\title{
Novel approach for mounting high power diode laser bars on passive copper heatsinks (Withdrawal notice)
}

Matthias Schroeder, Ekkehard Werner, Petra Hennig, Marco Koschorreck

Matthias Schroeder, Ekkehard Werner, Petra Hennig, Marco Koschorreck, "Novel approach for mounting high power diode laser bars on passive copper heatsinks (Withdrawal notice)," Proc. SPIE 11261, Components and Packaging for Laser Systems VI, 112610E (3 June 2020); doi: $10.1117 / 12.2546537$

SPIE. Event: SPIE LASE, 2020, San Francisco, California, United States 


\section{Novel approach for mounting high power diode laser bars on passive copper heatsinks (Withdrawal notice)}

Matthias Sc hroeder, Ekkehard Wemer, Petra Henning, Marco Koschorreck

J ENOPTIK Laser GmbH (Gemany)

https://www.spiedigitallibrary.org/conference-proceedings-of-spie/11261/112610E/Novel-approach-formounting-high-power-diode-laser-bars-on/10.1117/12.2546537.full

Event Photonic s West, Components and Packaging for La ser Systems VI, San Fra nc isc o, Califomia, United States 2020

Online Publication Date: 11 Ma rch 2020

Withdrawn from Publication: 3 J une 2020

Publisher's Note: This paper, originally published on 11 March 2020, was withdrawn per author request. 